\title{
Implicaciones de la reflexión y la mediación didáctica en docentes en formación durante su práctica educativa
}

\author{
Implications of reflection and didactic mediation in teachers in training during their \\ educational practice
}

\section{Implicações da reflexão e mediação didática em professores em formação durante sua prática educativa}

\author{
Edgar Andrés Espinosa Rios ${ }^{1}$ \\ Karen Dayana González López ${ }^{2}$ \\ Lizeth Tatiana Hernández Ramírez ${ }^{3}$
}

Recibido: junio de 2018

Aceptado: noviembre de 2018

Para citar este artículo: Espinosa-Rios, E. A., González-López, K. D. y Hernández-Ramírez, L. T. (2019). Implicaciones de la reflexión y la mediación didáctica en docentes en formación durante su práctica educativa. Revista Científica, 34(1), 101-122. Doi: https://doi.org/10.14483/23448350.13442

\section{Resumen}

El propósito de esta investigación fue indagar cómo los procesos reflexivos y los fundamentos de la mediación didáctica contribuyen a los procesos de enseñanza que realizan los docentes en formación en Ciencias Naturales durante su práctica educativa. Se trata de un estudio de caso en el que se diferencia cuatro momentos: la selección de la muestra de estudio, la formación teórico-práctica realizada por un docente mediador, la práctica educativa realizada por los docentes en formación con una intensidad de 25 horas y el análisis de las filmaciones de las clases y las entrevistas realizadas por los docentes en formación. Como resultado se observó la importancia de los procesos de reflexión y los fundamentos de la mediación didáctica en la formación docente y la necesidad de articular los conocimientos disciplinares, pedagógicos y didácticos desde contextos reales. El hecho de tener bases sólidas desde lo disciplinar no garantiza el buen desempeño, de ahí que los contextos reales se liguen a la reflexión sobre la acción en función de volver consciente las debilidades y fortalezas, desarrollando la capacidad de reflexión para mejorar los procesos de enseñanza, aprendizaje y evaluación.

Palabras clave: enseñanza, formación docente, práctica educativa.

\begin{abstract}
The purpose of this research is to investigate how the reflexive processes and the foundations of didactic mediation contribute to the teaching processes that teachers do in the formation of Natural Sciences during their educational practice. The
\end{abstract}


methodology is a case study with four moments. Selection of the study sample; The theoretical and practical training carried out by a mediating teacher; The educational practice carried out by teachers in training with an intensity of 25 hours; The analysis of the filming of the classes and the interviews carried out by the teachers in formation. As a result, it was observed the importance of reflection processes and the foundations of didactic mediation in teacher training and the need to articulate disciplinary, pedagogical and didactic knowledge from real contexts; the fact of having a solid foundation from the discipline does not guarantee the good performance, hence the real contexts are linked to the reflection on the action in order to make aware the weaknesses and strengths, developing the capacity for reflection to improve the teaching processes-learning-evaluation.

Keywords: Educational Practice, Teaching, Teacher Training.

\section{Resumo}

O objetivo desta pesquisa é investigar como os processos reflexivos e os fundamentos da mediação didática contribuem para os processos de ensino realizados pelos professores em formação em Ciências Naturais durante sua prática educativa. A metodologia é um estudo de caso com quatro momentos. A seleção da amostra do estudo; A formação teórico-prática realizada por um professor mediador; A prática educativa realizada pelos professores em treinamento com uma intensidade de 25 horas; A análise das filmagens das aulas e as entrevistas realizadas pelos professores em formação. Como resultado, observou-se a importância dos processos de reflexão e os fundamentos da mediação didática na formação de professores e a necessidade de articular conhecimentos disciplinares, pedagógicos e didáticos a partir de contextos reais; Tendo uma base sólida a partir da disciplina não garante bom desempenho, daí os contextos reais estão ligados à reflexão sobre a acção com base em re pontos fortes e fracos conscientes, desenvolvendo habilidades de pensamento para melhorar o ensino -aprendizagem-avaliação.

Palavras-chaves: ensino, formação de professores, prática educativa.

\section{Introducción}

La educación en ciencias naturales, según lo plantea Schwab (1971, como se cita en Espinosa, 2015), es una disciplina práctica condicionada por la teoría, lo cual se debe tener presente para desarrollar los conocimientos que convergen en el acto educativo. Por ello, es pertinente reconocer que durante el transcurso de su formación inicial y profesional el docente requiere de conocimientos tales como: el pedagógico, didáctico, disciplinar, práctico, etc. Estos conocimientos que se han venido nutriendo desde la formación escolar se fortalecen en la universidad y en diversos espacios. Estos se afianzan aún más en la medida en que se enfrentan a situaciones más complejas en contextos educativos reales $u$ otras experiencias de enseñanza no formal (no institucional), los cuales exigen desarrollar y fortalecer competencias, habilidades, destrezas y actitudes.

Los conocimientos (disciplinares, pedagógicos, didácticos, prácticos, etc.) junto con el compromiso social que se requiere asuman los docentes, pueden lograr que los procesos de enseñanza, aprendizaje y evaluación (E-A-E) contribuyan a la formación integral de los educandos. Ángulo (2010) plantea que para que el profesor tenga una práctica apropiada y provechosa debe tener una formación teórica bien consolidada en el campo disciplinar, apoyada de una formación pedagógica, didáctica y práctica que hace de él un profesional autónomo. Por ello, "su formación debe asentarse en la apropiación de los fundamentos disciplinares de las ciencias y en las elaboraciones que aporta la investigación en didáctica con miras a la utilización significativa del conocimiento al aprender a enseñar ciencias" (Ángulo, 2010, p. 70).

Dichos conocimientos que adquieren los docentes en formación convergen en los espacios de la práctica educativa. Por ende, se considera que entender el potencial formativo de la práctica implica reconocerla como fuente de conocimientos 
y aprendizajes situados en contextos reales, los cuales contribuyen a enriquecer el conocimiento práctico y las competencias que se requiere tengan los educadores para poder ejecutar la acción educativa, cada vez con mayor significado y calidad. Este conocimiento práctico del docente es el constructo que surge a partir de la experiencia de enseñanza, es decir, ese conocimiento que se genera desde "la acción de planear, desarrollar y evaluar la enseñanza de un tema específico" (Espinosa, 2015, p. 54), sorteando los diversos inconvenientes que se pueden presentar en su práctica.

En simultáneo, se requiere sostener la convicción de que los docentes en formación que se enfrentan a la práctica educativa pueden fortalecer sus procesos de enseñanza a partir del diseño y la ejecución de propuestas educativas enfocadas en el paradigma de la mediación didáctica, las cuales buscan formar a los estudiantes en el conocimiento disciplinar. Pero estas también responden a las diversas necesidades educativas, entendiendo que al tratar con personas no se interactúa únicamente con conocimientos, también con las dimensiones socio-afectivas de los estudiantes y otros aspectos trascendentales que determinan el comportamiento en el aula de clases y sus procesos de aprendizajes.

A partir de lo anterior surge la necesidad de investigar cómo los procesos reflexivos y los fundamentos de la mediación didáctica contribuyen a los procesos de enseñanza que realizan los docentes en formación de Ciencias Naturales durante su práctica educativa. En este sentido, es indispensable analizar y reflexionar sobre la acción educativa de los docentes en formación y reconocer las diversas dificultades que presentan durante el desarrollo de la práctica, integrar los fundamentos de la mediación didáctica en el diseño y la ejecución de la práctica educativa a partir de procesos de reflexión e identificar los logros que pudieron alcanzar los docentes en formación al realizar el seguimiento de su práctica educativa en la cual se perfilaron como mediadores didácticos.

\section{Marco teórico}

\section{La práctica educativa, una experiencia de ense- ñanza-aprendizaje para la construcción del cono- cimiento de docentes en formación}

La práctica educativa es un espacio trascendental para la formación de los futuros educadores, aunque su noción sobre el quehacer del profesor está permeado en un primer momento por sus primeras experiencias como estudiantes de escuela, estas nociones se comienzan a transformar durante su formación teórica en la universidad y se fortalecen cuando se enfrentan nuevamente a la escuela, pero en esta ocasión desde el rol docente. De acuerdo con esto, la relación escuela-universidad es vital en la formación de los futuros licenciados (Hernández, Quezada y Venegas, 2016.

Espinosa (2015) plantea que Las experiencias no se deben limitar a una mera actividad en busca de un conocimiento, sino que en ellas ocurre la interrelación entre el individuo, en este caso el profesor en formación, con el medio ambiente físico-social en que desarrollará su profesión, es decir, la escuela y la comunidad educativa.

Esta interacción implica el análisis, el desarrollo, además de la reflexión de sucesiones de acciones e impresiones sobre la teoría y práctica educativas, basadas en conexiones con el contexto y las relaciones condicionantes entre las experiencias pasadas, presentes y futuras, haciendo posible la creación de significados colectivos acerca de la enseñanza y el aprendizaje Dewey, (2004).(Espinosa, 2015, p. 92)

Por ende, se reconoce que la práctica educativa de los docentes en formación es un proceso que permite que los futuros profesores puedan empezar a "construir racionamientos y acciones pedagógicas inteligentes que medien el diseño, desarrollo y evaluación de la enseñanza de un tema específico" (Espinosa, 2015, p. 65). Se reconoce que el proceso de formar profesores para que aprendan a enseñar de manera efectiva sobrepasa 
la transferencia o transmisión de conocimientos teóricos producto de la investigación sobre la enseñanza-aprendizaje (E-A). Se trata de proveer las condiciones necesarias para la construcción de conocimientos prácticos desde las experiencias de enseñanza en contextos reales. De hecho, gracias a estos últimos, considerados como conocimientos en la acción, integrados, heterogéneos e informados por la teoría, los profesores realmente adquieren la capacidad de solucionar los problemas singulares y contingentes típicos de su labor (Elbaz, 1981).

\section{La importancia de la reflexión sobre la acción en la práctica docente}

En la práctica educativa ocurren diversas situaciones que conllevan a una constante interacción entre los individuos que participan en ella. Dichas interacciones no son posibles premeditarlas debido a sus propias dinámicas. En palabras de Schön (1998, p. 26) "Las situaciones de la práctica no constituyen problemas que han de ser resueltos, sino situaciones problemáticas caracterizadas por la incertidumbre, el desorden y la indeterminación". Para responder a esas características de la práctica es indispensable que el profesional, en este caso el educador, reconozca que su labor no se resume en aplicar una receta, es algo que trasciende a la racionalidad técnica si el objetivo es formar verdaderamente personas íntegras explotando al máximo sus capacidades cognitivas. En este sentido, de acuerdo con Ríos:

La actividad docente debe trascender del simple hecho de implementar ciertas estrategias en el aula de clase, debe visualizarse como una actividad que invite a la reflexión, de tal forma que se haga consciente el papel que desempeña el docente en el aula y no simplemente entrar en un activismo que en gran medida no fomenta el desarrollo de una autonomía por parte de los educandos y por ende no contribuye al desarrollo de habilidades cognitivas. (2016, p. 12)

\section{Un acercamiento conceptual a la mediación didáctica}

Etimológicamente, la palabra "mediar" proviene del latín mediare, cuyo significado evoca una articulación entre dos entidades o dos términos en el centro de un proceso dialéctico o en un razonamiento (Escobar, 2011). En el ámbito educativo, este término se integra a partir de los trabajos desarrollados por Vigotsky, "quien considera que los procesos mentales superiores en los seres humanos son mediados por herramientas poderosas como el lenguaje, el mundo simbólico y el manejo de códigos" (Escobar, 2011, p. 59). Este autor considera que el aprendizaje es un proceso fundamentalmente social, por lo que la enseñanza constituye el medio por el cual progresa el desarrollo. Según lo plantea Pilonieta (como se cita en Escobar, 2011), la mediación se refiere a la forma de interacción pedagógica que conduce a la generación de experiencias positivas de aprendizaje, principal dispositivo de aprendizaje y desarrollo emocional de las personas.

Por su parte, Tébar (como se cita en Escobar, 2011), afirma que mediar es un estilo de interacción educativa, orientado por una serie de creencias y principios antropológicos y psicopedagógicos, el cual posibilita el reencuentro, la aceptación e implicación en un proceso transformador, modificador y constructor de la persona para llegar a descubrir la esencia de sí mismo y la esencia de las cosas. Asimismo, este autor asume que la mediación es una forma de interacción que abarca todos los ámbitos de la vida de los educandos y responde a todas sus necesidades afectivas, cognitivas, y sociales en función de superar el fracaso escolar, las dificultades de aprendizaje, la desmotivación de los alumnos y demás problemáticas educativas que inciden de manera negativa en los procesos de E-A-E.

La mediación didáctica no debe ser entendida únicamente como la interacción entre docente-estudiante y los conocimientos que ambos sujetos poseen y convergen en el acto educativo en 
función de lograr la construcción de conocimiento científico escolar. También se integra una acción humanizadora, positiva, constructiva y potenciadora de los estudiantes y los docentes que tiene lugar en el complejo mundo de la relación educativa (Tébar, 2009). Este concepto polisémico de enorme riqueza acoge valores como la confianza, la paciencia, el amor; conoce a las personas, a los entornos que las rodean a sus necesidades y a su vida. Su objetivo es la construcción y el desarrollo de diferentes habilidades en el estudiante, buscando así el desarrollo de su autonomía.

De igual forma, la mediación didáctica comprende, da significado y sentido; ayuda con su total disponibilidad y cooperación; motiva para provocar el éxito; potencia, interioriza y eleva los niveles de abstracción. Además, provoca un constante cuestionamiento para desafiar y lograr un cambio en los docentes y en los estudiantes. Asimismo, orienta, guía, organiza y planifica nuevas estrategias que se integran en los procesos educativos; a la vez autoevalúa, valora, critica, relaciona y estructura la multidisciplinariedad entre los conocimientos que son útiles y necesarios para que sean enseñados por los docentes y aprendidos por los educandos; logrando así la formación en actitudes, normas y valores, para fomentar la autonomía y la aceptación en los participantes del acto educativo (Tébar, 2009).

Por tanto, la medición concebida desde un contexto educativo debe involucrar al educador o toda persona que contribuya al desarrollo de los procesos de los estudiantes, el cual deberá convertirse en un intermediario entre el estudiante y el conocimiento, entre el estudiante y su entorno, entre el estudiante y sus pares (Espinosa, 2016).

\section{El uso del signo y del lenguaje en los procesos de mediación}

Según Hernández (1997), el problema que existe entre el sujeto y el objeto de conocimiento se soluciona a partir de una interacción dialéctica debida a la exposición a instrumentos socioculturales (herramientas y signos). Dicha relación se logra gracias a la actividad mediada, pues es a través de la interacción del individuo (estudiante) con su contexto sociocultural y con otro que promueva su desarrollo que él logra construir e internalizar las funciones psicológicas superiores (operaciones mentales) y la consciencia.

La mediación, como proceso trascendental para el desarrollo integral y cognitivo del estudiante, no es posible sin elementos como el lenguaje y los signos. Aunque el hombre está expuesto directamente a señales (no intencionadas), estas se convierten en signos. En palabras de Gutiérrez, Ball y Márquez: "desde el momento en que no se remiten a ser meras señales naturales aprehendidas por la experiencia, sino por señales no naturales, inventadas intencionalmente" (2008, p. 630). En este sentido el signo cobra significado al ser adaptado para presentarlo a otro con la finalidad de motivar alguna respuesta que regule su comportamiento.

Por otra parte, el hecho de emitir un signo a otra persona no implica que esta lo reciba y presente una respuesta satisfactoria ante el mismo. Esto, pues según Gutiérrez, Ball y Márquez (2008) el signo también exige que la persona hacia la que va dirigido tenga una elaboración cognitiva previa que le permita comprenderlo e interpretarlo. En este proceso de interacción entra la emisión y la respuesta a estímulos, el lenguaje es muy importante ya que se convierte en un "sistema de mediación simbólica". Para Ríos (2016), este elemento es vital para la construcción de significado, pues a través de este se representan los signos en diversas prácticas sociales (en este caso la educación) que le permiten a la persona entender su realidad y actuar ante ella.

En este sentido, la mediación se torna un proceso que depende de los signos o estímulos que el profesor (mediador) selecciona, adapta y presenta al estudiante de forma cuidadosa y dotados de significado, utilizando el lenguaje apropiado que le permita comprenderlo y usarlo para responder a los sucesos de su cotidianidad. 


\section{Metodología}

El enfoque metodológico empleado para el desarrollo de la presente investigación es el estudio de caso, el cual en palabras Stake (2005) se puede definir como: "el estudio de la particularidad y de la complejidad de un caso singular, para llegar a comprender su actividad en circunstancias importantes [...] su objetivo básico es comprender el significado de una experiencia" (p. 16). Además, autores como Hernández, Fernández y Baptista (2006) plantean que el estudio de casos permite emplear métodos cuantitativos, cualitativos o mixto.

Teniendo en cuenta lo anterior, se reconoce que para poder dar solución a la pregunta de investigación (¿cómo los fundamentos de la mediación didáctica contribuyen a los procesos de enseñanza que realizan los docentes en formación de Ciencias Naturales durante su práctica educativa?), se explican a continuación los instrumentos empleados, la población objeto de investigación y la metodología desarrollada, la cual se realiza a partir de cuatro momentos.

\section{Instrumentos empleados}

Se emplearon tres instrumentos durante el desarroIlo de la investigación. El primero es el cuestionario de indagación de concepciones teórico-prácticas sobre la E-A-E en profesores en formación (anexo $1)$, con el cual se indagaron las concepciones teóricas que los docentes en formación (DF) poseen. Dicho instrumento hace parte de las herramientas que se emplean en el curso de mediación didáctica ofrecido a estudiantes de pregrado de la Universidad del Valle. El segundo y tercer instrumento son una tabla de valoración y una rejilla de análisis de clase, instrumentos empleados para la valoración y análisis de las clases realizadas por los DF.

\section{Población}

Participaron dos estudiantes pertenecientes a la Licenciatura en Educación Básica con énfasis en
Ciencias Naturales y Educación Ambiental de la Universidad del Valle de séptimo semestre, con un nivel de formación disciplinar y pedagógico de la carrera correspondiente a más del $80 \%$ de la estructura curricular de la licenciatura. Es de anotar que el simple hecho que los estudiantes hayan cursado y aprobado de buena forma cada una de las asignaturas de la estructura curricular del programa no significa que tengan el mismo nivel de apropiación del conocimiento, pero da garantía de que tienen una base conceptual similar.

\section{Desarrollo del diseño metodológico}

\section{Primer momento}

Este corresponde a la selección de la muestra, la cual se realizó con voluntarios. La vinculación en la investigación implicó un seguimiento por dos semestres, tiempo en el cual los DF diseñaron la propuesta y la aplicaron. El desarrollo e implementación se realizó en el marco de la asignatura Investigación en el aula, la cual corresponde a la práctica educativa que realizan los estudiantes de la licenciatura en mención.

Para la caracterización de la muestra se aplicó un cuestionario (anexo 1), con el cual se indagaron las concepciones que tienen los DF sobre los procesos de $\mathrm{E}-\mathrm{A}-\mathrm{E}$, las etapas que consideran debe tener una clase de Ciencias Naturales y con qué tipo de actividades las desarrollaría; también se plantearon situaciones a las que se enfrentan normalmente los docentes para conocer cómo actúan los estudiantes ante ellas.

Posterior al cuestionario, los DF diseñaron e impartieron una clase a los compañeros y profesores, es decir, un proceso de microenseñanza. Según autores como Olvera y Gutiérrez es una técnica que "consiste en descomponer el proceso de enseñanza con base en la simulación en pequeñas unidades fáciles de entender y susceptibles de ser practicadas en una situación simulada lo más cerca posible a la realidad de un salón de clases" (2013, p. 3). Es decir, se busca que el DF 
desarrolle procesos de enseñanza con un grupo de estudiantes; esto es monitoreado y filmado con la intención de realizar un análisis sobre la actuación e interacción del docente. Algunos aspectos que se analizan en los procesos de microenseñanza ${ }^{4}$ de acuerdo a la Stanford (Ortuño, s.f.) son: claridad de objetivos, pertinencia de ellos, selección y organización de contenido adecuado, forma en la cual inicia la clase, claridad de exposición, dinámicas de la clase, participación y atención de los estudiantes, selección de materiales, cierre de la clase, variedad de procedimientos para evaluar, uso de evaluación para mejorar la enseñanza y complementos y comunicación.

Después del proceso de microenseñanza, los estudiantes diseñaron y aplicaron una clase en una institución educativa. La temática a enseñar debía corresponder con el cronograma de la institución (ya no es de libre elección como en el proceso de microenseñanza), cuyo tiempo de desarrollo oscilaba entre 1 y 2 horas, las cuales se filmaron para un análisis posterior a partir de "la rejilla de análisis de clase" (véase anexo 3).

Con el análisis del cuestionario inicial, el proceso de microenseñanza y las clases en un contexto real se caracterizó a los docentes en formación de acuerdo con sus concepciones teóricas y prácticas en los procesos de enseñanza, en el cual ellos se conciben como docentes.

\section{Segundo momento}

Considerando los resultados del cuestionario y las microclases, se diseñó y ejecutó un proceso de formación a través de seminarios que integran la teoría, la práctica y la reflexión como elementos trascendentales para la formación docente. De acuerdo a esto, se plantea la mediación didáctica como punto de integración de dichos elementos, con los cuales se pretende fortalecer a los docentes en formación en aquellas cualidades que, según

La microenseñanza hace referencia a clases que se dictan en corto tiempo (20 minutos) con poblaciones reducidas
Feuerstein (1996) y Tébar (2009), son indispensables para el trabajo en el aula con los estudiantes.

En las primeras sesiones de trabajo se realizó la observación de las clases de profesores expertos y no expertos, tanto en los seminarios de formación interna como en aquellas clases realizadas en contexto real. Posteriormente, se reflexionó a partir de lo observado en cada clase, teniendo en cuenta la rejilla comentada en el apartado anterior.

Dentro del proceso de formación docente se realizaron seminarios teórico-prácticos en los que se trataron los siguientes aspectos: ¿realmente estoy listo para ser docente?; la mediación didáctica en el aula de clase (generalidades); fundamentos del paradigma mediador; habilidades cognitivas I; habilidades cognitivas II; el perfil didáctico del profesor mediador; ¿cómo diseñar una clase?; la importancia de la reflexión en los procesos de formación docente.

\section{Tercer momento}

En este momento los docentes en formación llevaron a cabo la construcción y ejecución de la propuesta de E-A, la cual hace parte del marco de la práctica educativa que desarrollan los estudiantes de la licenciatura durante 25 horas en un contexto educativo real. La fundamentación del diseño y la ejecución de esta propuesta se enmarcan en lograr la construcción y el fortalecimiento de los conocimientos disciplinares, pedagógicos, didácticos y prácticos que deben caracterizar el ejercicio de los docentes. De igual forma, se resalta la importancia de los DF al poder aplicar los conocimientos de la mediación didáctica.

Durante este proceso, los DF contaron con el acompañamiento del docente mediador, el cual cooperó en el análisis de las propuestas que ellos realizaron, retroalimentándolas y contribuyendo a que esta experiencia de E-A fuese significativa y permitiese que los DF articulen la teoría y la práctica, de tal forma que los estudiantes logren la construcción del conocimiento científico escolar. 
Los DF realizaron procesos de reflexión antes, durante y después de su intervención en el aula, la cual se considera una fuente de conocimiento importante. Además, se hizo el registro fílmico de cuatro clases, cada una de ellas con una intensidad de dos horas; las filmaciones de las clases se distribuyeron a lo largo de las 25 horas que los DF emplearon en su práctica docente.

\section{Cuarto momento}

En este momento se evalúa el proceso de E-A que han venido desarrollando los DF. Es importante reconocer que la evaluación es de carácter formativo.

Se proponen tres dimensiones para evaluar la práctica educativa de los DF: 1) el pensamiento didáctico del profesor y la planificación de la enseñanza; 2) la interacción educativa dentro del aula; y 3) la reflexión sobre los resultados alcanzados, dimensiones que están relacionadas con los instrumentos empleados durante el desarrollo de la investigación. La relación entre estas tres dimensiones es interdependiente, es decir, cada una de ellas afecta y es afectada por las otras, por lo cual resulta indispensable abordarlas de manera integrada.

\section{Resultados}

A continuación, se presentan los resultados de los DF sobre las concepciones teórico-prácticas sobre la E-A-E y los resultados obtenidos del análisis de las clases realizadas (microclases realizadas como parte de los seminarios de formación docente, clase en contexto real). Los valores presentados son el resultado de la matriz implementada (anexo 3) y la escala utilizada (anexo 2) para asignar la categorización, respectivamente.

\section{Resultados de los DF sobre las concepciones teóri- co-prácticas sobre la E-A-E}

Se pudo establecer que, de acuerdo a su preparación académica, los DF en el momento tenían nociones teóricas para el diseño u organización de una clase. Sin embargo, se puede observar algunas debilidades conceptuales y contradicciones cuando se les presentan casos o problemáticas. Por ejemplo, el docente en formación 1 (DF1) considera que la evaluación es sumativa en lugar de formativa y constante. El docente en formación 2 (DF2) cree como un aspecto importante la evaluación y las ideas previas de los estudiantes, pero no incluye los contenidos científicos como algo importante para la enseñanza de las ciencias.

En general, los DF resaltaron aspectos en su mayoría de corte constructivista en la medida en que se evidencia su interés por tener presente las necesidades de los estudiantes, las prácticas evaluativas formativas y el contexto sociocultural como una herramienta esencial en sus estrategias de enseñanza.

\section{Resultados de microclases}

En la tabla 1 se presentan las valoraciones de cada una de las clases realizadas por los DF. Posteriormente, se muestra el análisis de los resultados obtenidos según la categoría.

Tabla 1. Valoraciones de las categorías de análisis microclases realizada por los DF (véase el anexo 2 "escalas de valoración").

\begin{tabular}{lcccc}
\hline Categoría & \multicolumn{2}{c}{ DF1 } & \multicolumn{2}{c}{ DF2 } \\
Proceso de planeación de clase & Clase & Valoración & Clase & Valoración[A30] \\
Desarrollo de los procesos de E-A-E & $9 / 40$ & PS & $9 / 40$ & PS \\
Aspectos motivacionales de la clase & $53 / 130$ & AS & $60 / 130$ & AS \\
\hline
\end{tabular}

Fuente: Elaboración propia de los autores. 


\section{Categoría: proceso de planeación de clase}

DF1 y DF2 presentan resultados parcialmente satisfechos (PS). Según la rejilla de análisis, se entiende como dificultades en el proceso de planeación de la clase, no tienen en cuenta los estándares que propone el Ministerio de Educación Nacional (2014). No se evidencia en ellos de forma clara los fundamentos pedagógicos y didácticos. Considerando los resultados del cuestionario 1, las teorías y modelos que ellos expresan no se reflejan en el ejercicio de planeación, colocando en duda su nivel de apropiación de estos.

\section{Categoría: desarrollo de los procesos de E-A-E}

DF1 y DF2 presentan resultados algo satisfechos (AS) y, teniendo en cuenta la rejilla de análisis, se interpreta como bajo desempeño, lo cual se refleja en aspectos como: establecer relaciones entre la temática a enseñar y la cotidianidad o realidad de los estudiantes para que fuera más significativo el conocimiento, el uso inadecuado o la escasez de estrategias metodológicas, la no indagación en los estudiantes con el objetivo de reconocer posibles dificultades de aprendizaje o problemas que puedan interferir en su proceso cognitivo y baja integración de las dimensiones conceptuales, procedimentales y actitudinales.

\section{Categoría: aspectos motivacionales de la clase}

DF1 y DF2 obtuvieron resultado parcialmente satisfecho (PS) y a partir de la rejilla de análisis se infiere que no tienen en cuenta las respuestas o aportes realizados por los estudiantes. DF1 y DF2 no motivan a los estudiantes a trabajar de forma colaborativa y se estimula muy poco el interés y la curiosidad por la temática que enseñan.

\section{Resultados de la primera clase en un contexto real (clase Yumbo)}

En la tabla 2 se presentan los resultados de DF1 y DF2 de la clase realizada en una institución educativa del municipio de Yumbo, Valle del Cuaca (Colombia).

Tabla 2. Valoraciones de las categorías de la clase realizada por los DF en Yumbo.

\begin{tabular}{|c|c|c|c|c|}
\hline & DF1 & & DF2 & \\
\hline Categoría & Clase & Valoración & Clase & Valoración \\
\hline $\begin{array}{l}\text { Proceso de } \\
\text { planeación de } \\
\text { clase }\end{array}$ & $9 / 40$ & PS & $26 / 40$ & $\mathrm{~S}$ \\
\hline $\begin{array}{l}\text { Desarrollo de los } \\
\text { procesos de E-A-E }\end{array}$ & $57 / 130$ & AS & $81 / 135$ & AS \\
\hline $\begin{array}{l}\text { Aspectos } \\
\text { motivacionales de } \\
\text { la clase }\end{array}$ & $8 / 25$ & PS & $10 / 25$ & PS \\
\hline
\end{tabular}

Fuente: Elaboración propia de los autores.

\section{Resultados de las clases-práctica educativa DF1}

En la tabla 3 se presentan los resultados de las clases realizadas por DF1 en la práctica educativa.

\section{Resultados de las clases práctica educativa DF2}

En la tabla 4 se presentan los resultados de las clases realizadas por DF2 en la práctica educativa.

Tabla 3. Valoraciones de las categorías de la clase realizada por los DF en la práctica educativa.

\begin{tabular}{lllllllll}
\hline Categoría & Clase 1 & Val. & Clase 2 & Val. & Clase 3 & Val. & Clase 4 & Val. \\
Proceso de planeación de clase & $13 / 40$ & PS & $16 / 40$ & PS & $25 / 40$ & S & $26 / 40$ & S \\
Desarrollo de los procesos de E-A-E & $41 / 130$ & PS & $52 / 130$ & PS & $55 / 135$ & AS & $67 / 135$ & AS \\
Aspectos motivacionales de la clase & $6 / 25$ & PS & $10 / 25$ & PS & $12 / 25$ & AS & $13 / 25$ & AS \\
\hline
\end{tabular}

Fuente: Elaboración propia de los autores. 
Tabla 4. Valoraciones de las categorías de la clase realizada por los DF en la práctica educativa.

\begin{tabular}{lllllllll}
\hline Categoría & Clase 1 & Val. & Clase 2 & Val. & Clase 3 & Val. & Clase 4 & Val. \\
Proceso de planeación de clase & $21 / 40$ & PS & $24 / 40$ & AS & $23 / 40$ & AS & $23 / 40$ & AS \\
Desarrollo de los procesos de E-A-E & $57 / 135$ & PS & $75 / 135$ & AS & $84 / 135$ & S & $82 / 135$ & S \\
Aspectos motivacionales de la clase & $8 / 10$ & PS & $15 / 25$ & AS & $13 / 25$ & AS & $14 / 25$ & AS \\
\hline
\end{tabular}

Fuente: Elaboración propia de los autores.

\section{Análisis de la primera clase en un contexto real (clase Yumbo)}

\section{Proceso de planeación de clase}

Se videncia que las actividades que se plantean están orientadas para entender un tema en particular, pero no se parte de las posibles dificultades de aprendizajes que los estudiantes presentan. No se observa claramente que el DF emplee actividades para motivar o introducir a los estudiantes en la temática a desarrollar; esto resulta en contravía del supuesto según el cual los DF manifiestan tener claros los referentes teóricos constructivistas, aspecto que se confirma fuertemente en DF1. Este realiza una planeación muy parecida a la microclase, plantea un párrafo que carece de un propósito, no hay relación con el estándar ni estrategias o herramientas a utilizar.

DF2 establece relación entre los propósitos, el estándar y las actividades propuestas encaminadas a fortalecer y desarrollar en los estudiantes las diversas habilidades y competencias que considera importantes de acuerdo al núcleo conceptual que se trabajó. Asimismo, incluye el componente descriptivo que relata de manera más específica los momentos en los cuales se desarrolla la clase. De igual forma, se destaca que la planeación de las actividades se caracteriza por tener un evidente propósito de despertar un mayor interés en los estudiantes.

\section{Desarrollo de los procesos de E-A-E}

Ambos DF se caracterizan por presentar diversas dificultades y debilidades en la fundamentación que debería sustentar toda acción del docente en el aula, ya sea disciplinar, didáctica o pedagógica. En el caso de DF1, su fortaleza radica en el conocimiento disciplinar que sustenta su acción en el aula. Para DF2 este conocimiento es una de las dificultades que presenta durante el desarrollo de su práctica educativa. Sin embargo, es importante reconocer que DF2 se caracteriza por articular de manera significativa y relevante sus conocimientos didácticos y pedagógicos. De igual forma, no asume una posición autoritaria, se preocupa por utilizar diversas estrategias o herramientas de enseñanza a partir de las cuales va guiando el proceso de aprendizaje.

\section{Aspectos motivacionales de la clase}

DF1 no incluyó en su práctica actividades que invitarán a los estudiantes a trabajar de forma cooperativa y colaborativa en función de generar una dinámica de E-A. Asimismo, la motivación de los estudiantes durante la práctica del docente es escasa.

DF2 promueve el trabajo cooperativo y colaborativo, se enfoca en que los estudiantes participen y aporten sus ideas para construir el conocimiento; sin embargo, no resalta en gran medida los aportes, las virtudes y las cualidades de los estudiantes. De igual forma, no logra animar a todos los estudiantes para que participen de manera activa, tampoco reconoce los elementos positivos de sus comentarios.

\section{Análisis de la clase 1 (práctica educativa)}

\section{Proceso de planeación de clase}

DF1 y DF2 presentan diversas falencias en plantear actividades que pueden despertar el interés 
en los estudiantes y motivarlos a participar y a generar comunidades de aprendizaje que contribuyan a fortalecer y desarrollar habilidades y destrezas en ellos, las cuales son más notorias en DF1 (según los resultados obtenidos). De igual forma, se reconoce que los propósitos que plantean se enfocan más en que el estudiante adquiera un conocimiento, pero no en promover el desarrollo de habilidades. Por otra parte, se identifica que DF1 no tiene en cuenta los estándares en el momento de realizar la planeación. DF2 sí incluye los estándares en su planificación, teniendo en cuenta el grado de escolaridad en el que se encuentran y el desarrollo cognitivo progresivo que los educandos irán fortaleciendo a partir del saber, el hacer $y$ el ser.

\section{Desarrollo de los procesos de E-A-E}

Los DF presentan ciertas debilidades en el manejo disciplinar de los estudiantes. La forma en que se muestra el contenido en ocasiones no despierta el interés y motivación. Las actividades que se proponen se enfocan más en repetir lo que hayan podido memorizar de los conceptos. Los estudiantes no identifican, analizan, argumentan o proponen relaciones de semejanza y diferencia entre algunas situaciones que sean del contexto cotidiano de los educandos, para promover habilidades y destrezas que aporten a la formación de su proyecto de vida, además del uso que se hace de algunas herramientas.

\section{Aspectos motivacionales de la clase}

Con respecto a los aspectos motivacionales de la clase que desarrolló DF1, no se identifica que el ambiente de aprendizaje genere gran motivación en los estudiantes, además de no propiciar el trabajo cooperativo y colaborativo.

DF2 resalta algunos aportes con frases como "muy bien", pero no se trasciende al análisis de lo que los estudiantes comentan, sino en obtener una respuesta exacta de la pregunta realizada.

\section{Análisis de clase 2 (práctica educativa)}

\section{Proceso de planeación de clase}

DF1 diseñó actividades para despertar el interés en los estudiantes y motivarlos a comprometerse más con su proceso de aprendizaje al implementar actividades en grupo que involucraba el desarrollo de habilidades lingüísticas como la lectura y la escritura, así como la realización de talleres que apoyaron el desarrollo de la clase de manera efectiva y orientada hacia los objetivos establecidos. La planeación promueve los conocimientos conceptuales y establece relaciones entre la ciencia y la sociedad. Sin embargo, no se tuvieron en cuenta los estándares y las dificultades de aprendizaje que se generan en los estudiantes al enfrentar estos contenidos.

DF2 tiene en cuenta los estándares, pero no plantea propósitos de forma específica. En cuanto a los aspectos descriptivos de las actividades, plantea cinco momentos en los cuales se desarrollaría la clase. Si bien en los momentos se evidencia una estructura clara de inicio, desarrollo y cierre, falta más especificidad en la descripción de lo que se realizará. Se sigue destacando de este DF la relación que establece entre los propósitos, el estándar y las actividades propuestas, encaminadas a fortalecer y desarrollar en los estudiantes las diversas habilidades y competencias.

\section{Desarrollo de los procesos de E-A-E}

Se evidencia que el manejo de grupo por parte de DF1 no contribuye a lograr un buen desarrollo de la clase y un ambiente de trabajo adecuado. Además, el tono de su voz no fue el indicado para mostrar autoridad y lograr que los estudiantes mejoren su comportamiento.

La actividad de DF2 se mantuvo mucho más organizada, tuvo en cuenta la necesidad de construir ante los estudiantes una imagen de autoridad, el lenguaje del docente es claro. Aunque se evidencia que falta mayor manejo disciplinar, se observó interacción entre los estudiantes. 


\section{Aspectos motivacionales de la clase}

DF1 resalta los aportes que realizan los estudiantes y los anima a participar de forma voluntaria. Sin embargo, no trasciende en reconocer las virtudes y cualidades que caracterizan a los estudiantes para así motivar aún más su participación.

DF2 resalta algunos aportes de los estudiantes, pero no se observa que lo realice con la intención de promover en ellos mayor participación o de verdaderamente resaltar sus comentarios o habilidades. En la realización de la actividad se fomentó el trabajo en equipo.

\section{Análisis de clase 3 (práctica educativa) \\ Proceso de planeación de clase}

DF1 planteó una actividad experimental con la cual despertó el interés y la motivación en los estudiantes. Sin embargo, no se tuvo en cuenta los estándares. Se evidencia coherencia entre lo que se planeó y los objetivos que se deseaban lograr.

DF2 mantuvo la misma estructura de planeación de clase, planteó actividades en las que consideró el gusto de los estudiantes por el uso de los computadores integrándolo a sus estrategias. No obstante, las actividades no exigen mayor nivel de abstracción o interiorización.

\section{Desarrollo de los procesos de E-A-E}

DF1 sigue presentando ciertas debilidades en relación al manejo de grupo y la disciplina necesaria para poder generar un ambiente adecuado de enseñanza. Se destaca el esfuerzo que realiza el docente para lograr que los estudiantes establezcan relaciones entre los conocimientos abordados en clases anteriores y los que se trabajarán posteriormente. Todavía se presentan dificultades frente: al manejo del grupo, identificación de las dificultades de los estudiantes, el cierre de la clase, integrar los contenidos conceptuales, procedimentales y actitudinales del conocimiento científico y establecer una interacción constante entre docente-estudiante y estudiante-estudiante.

DF2 ha mejorado en el manejo de grupo. Pero, al inicio de la clase presentó algunos inconvenientes para convocar a todos los estudiantes y llamar su atención. Un aspecto a resaltar del DF es que desde el inicio de sus clases se ha observado una secuencia clara en la que se establecieron relaciones con lo abordado en las clases anteriores, ya que administra bien el tiempo.

\section{Aspectos motivacionales de la clase}

DF1 resalta los aportes que realizaban los estudiantes, pero lo hace de forma muy general para todo el grupo.

DF2 logra despertar el interés y la motivación en los estudiantes al integrar al desarrollo de la clase actividades que requieren el trabajo en grupo de forma cooperativa y colaborativa, así como el uso de recursos tecnológicos que Ilaman su atención y logran despertar su interés, curiosidad y expectativas por aprender.

\section{Análisis de clase 4 (práctica educativa)}

\section{Proceso de planeación de clase}

DF1 presenta coherencia entre los propósitos y la actividad. Por otra parte, como es una actividad evaluativa, con ella se busca hacer seguimiento del proceso de aprendizaje de los estudiantes e identificar posibles dificultades. Con la actividad planteada también se observa la necesidad de que los estudiantes interactúen y trabajen en equipo, aspecto que promueve la comunicación entre ellos y habilidades como el argumentar. Se evidencia el uso de herramientas tecnológicas como el video beam.

DF2 termina su práctica manteniendo la misma estructura en su planeación. La actividad de conclusión evoca lo trabajado en sesiones anteriores. Se destaca que la actividad propuesta exige un nivel de abstracción o interiorización mayor 
al promover en los estudiantes la búsqueda de soluciones para un problema ambiental. Se sigue evidenciado que al DF le falta integrar en sus actividades estrategias que le permitan a los estudiantes superar las dificultades conceptuales respecto al tema.

\section{Desarrollo de los procesos de E-A-E}

DF1 presenta mayor manejo del grupo que en la primera parte de la clase. El lenguaje utilizado para dar la instrucción es claro, pero a su voz le falta mayor proyección. Por otra parte, la autoridad del DF ha mejorado y aunque se observa que intenta mantener un buen ambiente para el aprendizaje, se evidencia la falta de estrategias para lograr atraer la atención de los estudiantes. En cuanto al manejo disciplinar esto ha sido un fuerte del DF; de igual forma se observa una buena conducción del tema y seguridad cuando habla.

DF2 siempre mantuvo un hilo conector entre las sesiones de clase. Se evidenció que al DF aún le falta mayor manejo disciplinar. Hay claridad en las secuencias de la clase. Por otra parte, aunque la autoridad del profesor mejoró, aún se siguen presentando inconvenientes en cuanto a la disciplina durante la clase, especialmente al inicio de la misma.

\section{Aspectos motivacionales de la clase}

En relación con los aspectos motivacionales, se analiza que ambos docentes se esfuerzan en poder llamar la atención de los estudiantes para motivarlos a participar activamente. Sin embargo, en algunas ocasiones las estrategias que se implementan en las clases no logran que todos los estudiantes se interesen en contribuir al desarrollo de la misma.

\section{Conclusiones}

Al integrar los procesos de mediación didáctica y la reflexión en la formación docente antes y durante la práctica educativa de los DF, se evidenció un importante desarrollo y fortalecimiento en la toma de conciencia sobre las decisiones que se implementaron en las actividades y las dinámicas surgidas en el aula de clase durante los procesos de enseñanza que no se resuelven únicamente con el conocimiento disciplinar.

Asimismo, reconocen la importancia de fortalecer diversas habilidades como las comunicativas y el manejo de grupo; de tal forma que se facilita la construcción de un ambiente de aprendizaje propicio para el aprendizaje de los estudiantes. Se evidenció que, ante el primer acercamiento a la práctica por medio de las microclases hasta finalizar el proceso de su práctica educativa en la escuela, se logró un importante progreso en relación a la intencionalidad, el significado y la trascendencia de las clases de los DF. Incluso se percibió en algunos casos indicios de cambios ante su imagen como docente y los referentes prácticos que tienen de enseñanza.

Se infiere que la práctica de los DF está influenciada en gran manera por su carácter, gustos personales y la apropiación del constructo teórico que han formado desde sus años de escolaridad y universitarios acerca de la educación y los procesos de E-A. Teniendo en cuenta esto, se ratifica la necesidad de que los docentes en formación visualicen de forma estructurada las teorías pedagógico-didácticas que sustenta sus acciones y que analicen las características que debe tener un docente independientemente de su personalidad y cuya línea de acción se enmarque en el constructivismo. De esta forma, se trasciende el momento de diseñar y realizar una clase del simple hecho de pensar (el docente) en cómo le gustaría que le enseñaran a reflexionar en lo que necesitan los estudiantes para apropiar el conocimiento.

Con esta investigación se confirma lo planteado en investigaciones realizadas por Espinosa(2015) y Espinosa (2015 y 2016), a partir de las cuales concluyeron la necesidad de que los docentes en formación se enfrenten a espacios en los que tengan la oportunidad de actuar en contextos reales y poner en juego los conocimientos adquiridos durante su formación. Sin embargo, este proceso no 
deben realizarlo de manera individual. Es trascendental contar con el acompañamiento constante de un docente experto el cual amplíe su perspectiva acerca de la reflexión sobre sus acciones y de lo que implica formar integralmente a seres humanos con diversos talentos, capacidades, dificultades, necesidades, competencias y así promover en ellos la construcción de un conocimiento científico escolar. De esta forma, se vuelven más conscientes de los procesos de mediación que realizan en su cotidianidad y se comienza a superar las dificultades halladas al inicio sobre la falta de coherencia entre el discurso, las acciones y las tendencias positivistas de las mismas.

La reflexión sobre la acción permite que los DF reconstruyan su concepción sobre la educación, la disciplina, la enseñanza, aprendizaje y la evaluación, sobre los ideales educativos con los cuales iniciaron y la realidad que se desarrolla en la escuela, aportándoles herramientas para analizar las implicaciones de sus hechos durante y después de su accionar en el aula de clase.

\section{Referencias}

Elbaz, F. (1981). The teacher's "practical knowledge": Report of a case study. Curriculum inquiry, 11(1), 43-71.

Escobar, N. (2011). La mediación del aprendizaje en la escuela. Accion Pedagógica, 20(1), 58-73.

Espinosa, T. (2015). La reflexión en la práctica docente: un medio para la construcción del conocimiento práctico de profesores de ciencias en formación inicial (tesis de maestría). Maestría en Educación con Énfasis en Enseñanza de las Ciencias, Universidad del Valle, Cali.

Espinosa-Ríos, E. A. (2016). La formación docente en los procesos de mediación didáctica. Praxis, 12(1), 90-102. DOI: https://doi. org/10.21676/23897856.1850

Feuerstein, R. (1996). La teoría de la modificabilidad estructural cognitiva. Educación Cognitiva, 1, 31-75.
Gutiérrez, M. E., Ball Vargas, M. S. y Márquez, E. (2008). Signo, significado e intersubjetividad: una mirada cultural. Educere, 12(43), 689-695.

Hernández, G. (1997). Módulo fundamentos del desarrollo de la tecnología educativa (bases psicopedagógicas). México: Ilce, OEA.

Hernández, M. P., Quezada, A. C. y Venegas, M. P. (2016). Análisis de la práctica docente en la formación inicial de profesores de religión. Educación y Educadores, 19(3), 357-369. DOI: https://doi.org/10.5294/edu.2016.19.3.3

Hernández, R., Fernández, C. y Baptista, P. (2006). Metodología de la investigación (Vol. III). México: McGraw-Hill.

Ministerio de Educación Nacional (2014). Lineamientos de calidad para las licenciaturas en educación (Programas de formación inicial de maestros). Bogotá: Ministerio de Educación Nacional.

Olvera, J. V. B. y Gutiérrez, M. A. C. (2013). Aplicando la técnica de microenseñanza en el modelo de aprendizaje basado en competencias (MABC). Eleventh LACCEI Latin American and Caribbean Conference for Engineering and Technology. Innovation in Engineering, Technology and Education for Competitiveness and Prosperity, Cancun, Mexico.

Ortuño, V. (sf). Microenseñanza, El Dr Vicente Ortuño resume en este artículo lo que es y lo que significa la microenseñanza dentro de las innovaciones educativas.

Ríos, E. A. E. (2016). La reflexión y la mediación didáctica como parte fundamental en la enseñanza de las ciencias: un caso particular en los procesos de la formación docente. TED: Tecné, Episteme y Didaxis, 40, 175-209.

Schön, D. A. y Coll Salvador, C. (1998). El profesional reflexivo: cómo piensan los profesionales cuando actúan. Barcelona: Paidós.

Stake, R. E. (2005). Investigación con estudio de caso. Madrid: Ediciones Morata.

Tébar, L. (2009). El profesor mediador del aprendizaje. Bogotá: Magisterio. 


\section{ANEXOS}

Anexo 1. Cuestionario de indagación de concepciones teórico-prácticas sobre la E-A-E en profesores en formación.

1. ¿Qué actividades o recursos emplearía para enseñar Ciencias Naturales?

2. ¿Cuáles serían las etapas que considera debe tener una clase de Ciencias?

3. De los siguientes principios, seleccione las cinco opciones que usted considere más importantes en la enseñanza de las Ciencias y organícelos teniendo en cuenta la siguiente escala: de mayor (1) a menor (5) grado de importancia.

- Conocer las ideas previas de los estudiantes.

- Plantear situaciones problemas.

- Los contenidos científicos.

- Evaluación permanente de los procesos de aprendizaje.

- El desarrollo de procesos experimentales.

- Elaboración de explicaciones del mundo natural.

- Otras, ¿cuáles?

4. ¿Cuáles son los conocimientos que debe tener un docente para llevar a cabo el proceso de E-A-E?

5. ¿Cuál es tu concepción del término pedagogía?

6. ¿Cuál es tu concepción del término didáctica?

7. ¿Qué entiendes por los conceptos de enseñanza, aprendizaje y evaluación?

8. En una institución educativa, los profesores de sexto grado consideran importante inculcar el sentido de la responsabilidad en los estudiantes y notan con preocupación que estos no están cumpliendo con las tareas escolares asignadas. Al preguntarles su opinión, los estudiantes consideran que para cumplir con las tareas se deben suspender las actividades extracurriculares obligatorias que ofrece la institución en la jornada complementaria porque estas demandan tiempo y obligaciones.

Para reforzar el sentido de responsabilidad en los estudiantes, los profesores proponen:

A. Exponer ante los padres de familia las ventajas de exigir a sus hijos el cumplimiento de las tareas escolares.

B. Considerar los compromisos que asumen los estudiantes en las dos jornadas para la asignación de tareas.

C. Premiar a los estudiantes que además de participar en ambas jornadas cumplen con las tareas académicas.

D. Privilegiar las tareas académicas que estimulen el intelecto porque las demás actividades son recreativas.

9. Durante el primer semestre de un año lectivo, un estudiante presenta bajo rendimiento académico en un área fundamental, en la que en años anteriores había sido sobresaliente. El estudiante muestra poco interés por el área, incumple con las tareas y a pesar de que los padres han sido convocados por el profesor para comunicarles la situación, su rendimiento es deficiente. Ante esta situación, el profesor, teniendo en cuenta su responsabilidad en el proceso educativo, decide que la mejor acción es: 
A. Indagar la causa del bajo rendimiento del estudiante en el área y hacer un diagnóstico de la situación.

B. Diseñar un programa de refuerzo para que el estudiante se nivele con los demás estudiantes.

C. Cambiar la metodología de enseñanza que viene utilizando para facilitar el aprendizaje del estudiante.

D. Concertar con el estudiante compromisos que incluyen acciones de seguimiento en el área.

Usted tiene a su cargo estudiantes de sexto y desea que a partir del siguiente problema respondan lo que ocurre con la temperatura en cada uno de los objetos cuando se introducen dos alambres, cada uno con temperatura de $50^{\circ} \mathrm{C}$ y $30^{\circ} \mathrm{C}$ en el recipiente con agua a $100^{\circ} \mathrm{C}$ de temperatura.

10. Al explorar las ideas previas de los estudiantes se halló que en su mayoría escogió la opción 3 que plantea que la temperatura sube a $180^{\circ} \mathrm{C}$ Sin embargo la respuesta correcta afirma que los objetos logran un equilibrio térmico, por esta razón usted decide:

A. Explicar el problema con ejercicios de lápiz y papel.

B. Registrar la temperatura de cada objeto después de intervalos de tiempo.

C. Confrontar la respuesta previa de los estudiantes con un experimento.

D. Hacer un experimento sencillo.

11. Si un estudiante es hábil para resolver memorísticamente problemas de química, pero al preguntarle por procesos de análisis y de interpretación no responde satisfactoriamente, entonces el maestro debe trabajar en sus estudiantes.

A. Ejercicios de análisis sobre su propia práctica de aprendizaje.

B. El enfrentamiento con situaciones problema en busca de respuestas aceptables.

C. Los pasos de resolución de problemas.

D. La sustentación de respuestas.

El problema central del maestro en el aula es la integración de su conocimiento con el conocimiento del estudiante, pues de la interpretación pedagógica que realice depende su actividad de enseñanza, aprendizaje y evaluación en el aula.

Considerando lo anterior frente al problema central de la evolución por Darwin y de los seis principios propuestos para solucionarlo, de los cuales, el primero afirma que, evolución significa cambio; el segundo plantea que los seres vivos tienen un ancestro común; el tercero dice que la evolución se explica por cambios graduales en el tiempo; el cuarto, por su parte, plantea que la selección natural es el mecanismo de la evolución; el quinto, que una población aislada geográficamente genera especies diferentes; y el sexto, que la adaptación es un proceso natural de los seres vivos.

12. Teniendo en cuenta lo anterior, la primera actividad educativa de clase que realizaría para enseñar la evolución sería

A. Explicar el origen de las especies según Darwin.

B. Explicar los seis principios de la evolución.

C. Conocer qué piensan los estudiantes sobre evolución.

D. Desarrollar un experimento previo sobre evolución. 
13. El examen es una práctica evaluativa muy común en el campo de la educación y en su desarrollo. Con los estudiantes se pueden evidenciar dos tendencias: La reducción a la reproducción de conceptos, para lo cual Pozo (1996) hace un llamado a los profesores para evitar que esto suceda; y el uso como instrumento de reflexión sobre los aprendizajes.

De acuerdo con lo anterior, las prácticas evaluativas diferentes del examen que favorecen la reflexión en los estudiantes son:

A. Trabajos elaborados en colectivo, elaboración de mapas conceptuales y resolución de cuestionarios cerrados.

B. Presentaciones orales argumentativas, elaboración de ensayos y revisión conjunta de programas de actividades.

C. Construcción de cuadros sinópticos, reportes de visitas de campo e informes escritos de un video.

D. Elaboración de mapas conceptuales, resolución de ejercicios y debates en torno a problemáticas.

14. Favorecer la formación de los estudiantes es una de las responsabilidades de todo profesor y para ello debe llevar a cabo una serie de acciones en las cuales se tenga en cuenta el carácter educable de los estudiantes. Un profesor desea implementar en su clase una serie de actividades que contribuyan a la formación de sus estudiantes. Una característica sustancial de estas actividades es que sean:

A. Dinámicas, a fin de motivar a los estudiantes para que concedan relevancia al aprendizaje de contenidos.

B. Cortas para mantener el interés de todos los estudiantes sobre un tema de estudio que afecta a la comunidad.

C. Próximas al contexto cultural y social de los estudiantes para integrarlas al ámbito escolar.

D. Coherentes con los objetivos procedimentales del área de estudio para articularlas con los estándares de competencias ciudadanas.

15. Los medios de comunicación masiva se han constituido en importante competidor de la escuela en la transmisión de valores sociales y morales. Frente a esta problemática se puede concluir que corresponde a la institución educativa

A. Ocuparse preferiblemente de la transmisión de conocimientos.

B. Permanecer ajena a los mensajes y valores que se trasmiten en los medios.

C. Analizar e integrar estas formas de educación a su práctica.

D. Contrarrestar los efectos nocivos con prácticas de convivencia. 
Anexo 2. Escalas de valoración.

\begin{tabular}{|l|l|}
\hline \multicolumn{2}{|l|}{$\begin{array}{l}\text { Valoración categoría } 1 \text { (planeación de } \\
\text { clase) }\end{array}$} \\
\hline $1-8$ & Insatisfecho (IS) \\
\hline $9-16$ & Parcialmente satisfecho (PS) \\
\hline $7-24$ & Algo satisfecho (AS) \\
\hline $25-32$ & Satisfecho (S) \\
\hline $33-40$ & Muy satisfecho (MS) \\
\hline
\end{tabular}

\begin{tabular}{|l|l|}
\hline \multicolumn{2}{|c|}{$\begin{array}{l}\text { Valoración categoría } 2 \text { (desarrollo de los } \\
\text { procesos de E-A-E) }\end{array}$} \\
\hline $1-27$ & Insatisfecho (IS) \\
\hline $28-54$ & $\begin{array}{l}\text { Parcialmente satisfecho } \\
\text { (PS) }\end{array}$ \\
\hline $55-81$ & Algo satisfecho (AS) \\
\hline $82-108$ & Satisfecho (S) \\
\hline $109-135$ & Muy satisfecho (MS) \\
\hline
\end{tabular}

\begin{tabular}{|l|l|}
\hline \multicolumn{2}{|l|}{$\begin{array}{l}\text { Valoración categoría } 3 \text { (aspectos } \\
\text { motivacionales de la clase) }\end{array}$} \\
\hline $1-5$ & Insatisfecho (IS) \\
\hline $6-10$ & Parcialmente satisfecho (PS) \\
\hline $11-15$ & Algo satisfecho (AS) \\
\hline $16-20$ & Satisfecho (S) \\
\hline $21-25$ & Muy satisfecho (MS) \\
\hline
\end{tabular}


Anexo 3. Rejilla de análisis de clase.

\begin{tabular}{|c|c|c|c|c|c|}
\hline $\begin{array}{l}\text { Aspecto a tener en cuenta } \\
\text { Valoración }\end{array}$ & Insatisfecho & $\begin{array}{l}\text { Parcialmente } \\
\text { satisfecho }\end{array}$ & $\begin{array}{l}\text { Algo } \\
\text { satisfecho }\end{array}$ & Satisfecho & $\begin{array}{l}\text { Muy } \\
\text { satisfecho }\end{array}$ \\
\hline Proceso de planeación de la clase & & & & & \\
\hline $\begin{array}{l}\text { El profesor plantea actividades pertinentes } \\
\text { que pueden despertar el interés en los } \\
\text { estudiantes para motivarlos. }\end{array}$ & & & & & \\
\hline $\begin{array}{l}\text { El profesor plantea propósitos pertinentes } \\
\text { que busquen promover el desarrollo de } \\
\text { habilidades y conocimientos. }\end{array}$ & & & & & \\
\hline $\begin{array}{l}\text { El profesor diseña y propone actividades } \\
\text { que exigen mayor nivel de abstracción e } \\
\text { interiorización, en función de potencializar } \\
\text { las capacidades de los alumnos. }\end{array}$ & & & & & \\
\hline $\begin{array}{l}\text { El profesor busca la información necesaria } \\
\text { para conocer las dificultades de aprendizaje } \\
\text { de los contenidos sus causas y efectos. }\end{array}$ & & & & & \\
\hline $\begin{array}{l}\text { El profesor incluye en su planificación } \\
\text { la utilización de diferentes estrategias } \\
\text { o herramientas tales como medios } \\
\text { audiovisuales, prácticos de laboratorio, } \\
\text { talleres que apoyan el desarrollo de la clase } \\
\text { de manera efectiva y orientada hacia los } \\
\text { objetivos establecidos. }\end{array}$ & & & & & \\
\hline $\begin{array}{l}\text { Se tiene en cuenta los estándares en el } \\
\text { momento de realizar la planeación. }\end{array}$ & & & & & \\
\hline $\begin{array}{l}\text { Las herramientas que se proponen son las } \\
\text { más adecuadas para alcanzar los objetivos } \\
\text { deseados. }\end{array}$ & & & & & \\
\hline $\begin{array}{l}\text { Se observa coherencia entre lo planeado y } \\
\text { los objetivos que se desean lograr. }\end{array}$ & & & & & \\
\hline Desarrollo de los procesos de E-A-E & & & & & \\
\hline
\end{tabular}




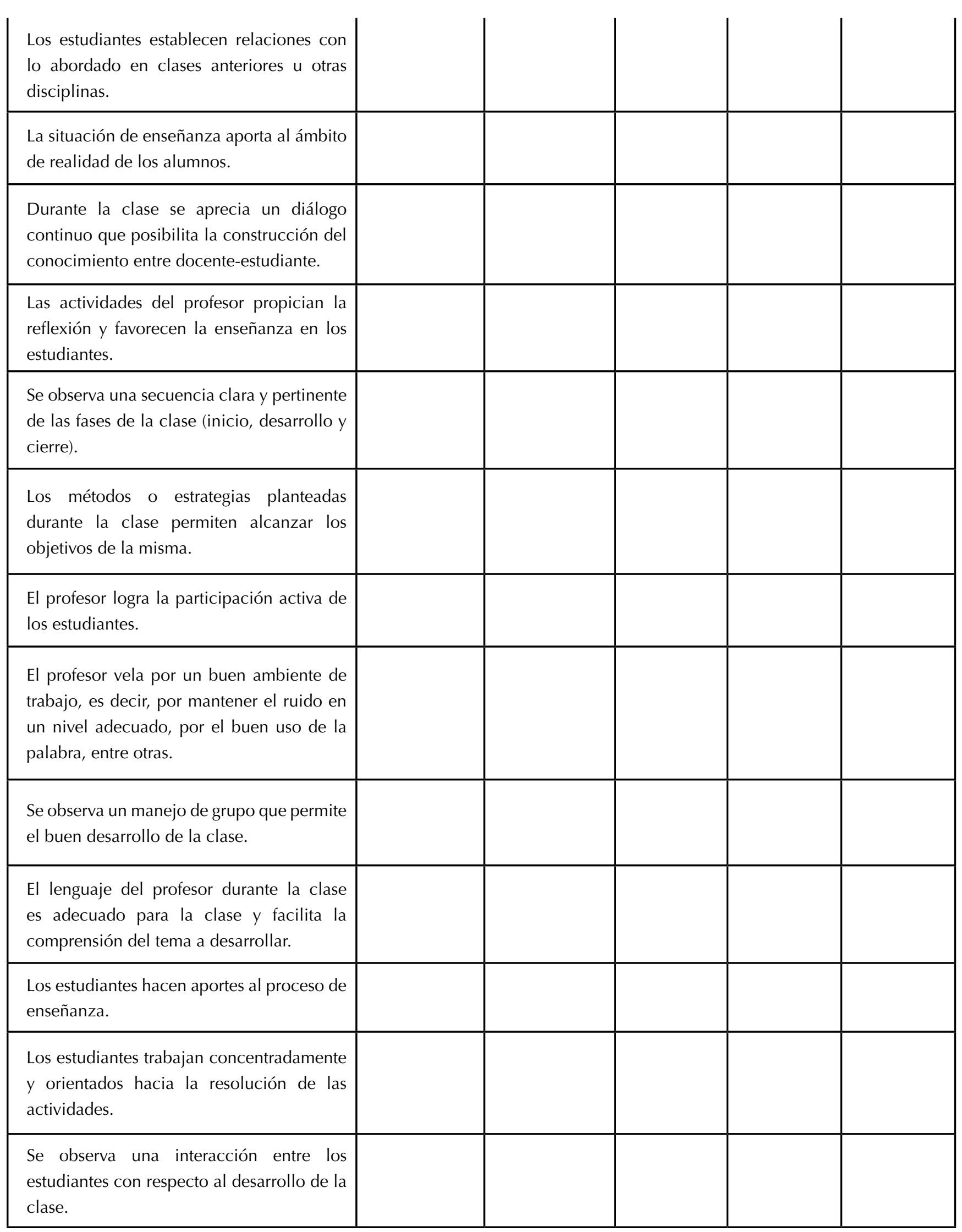




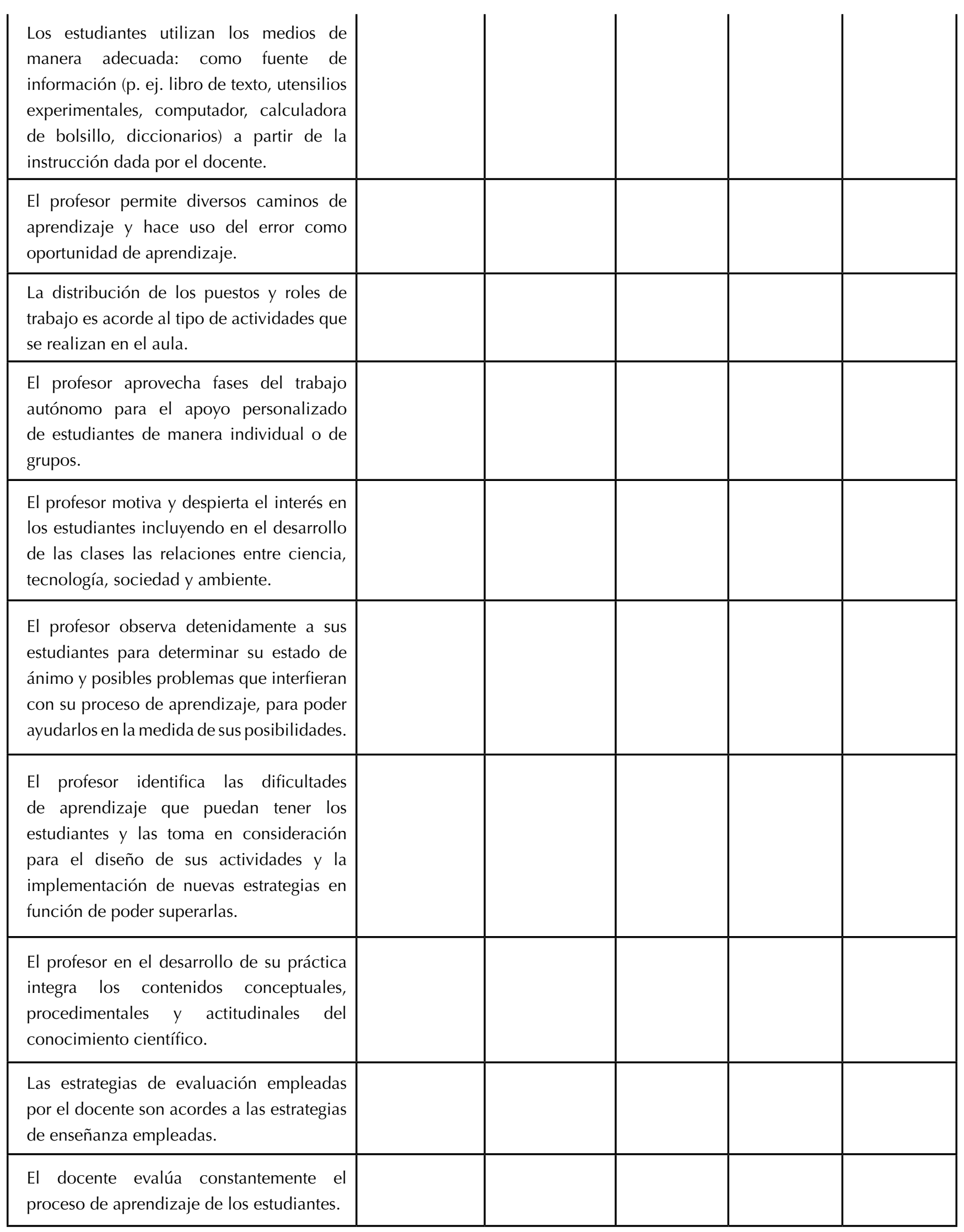




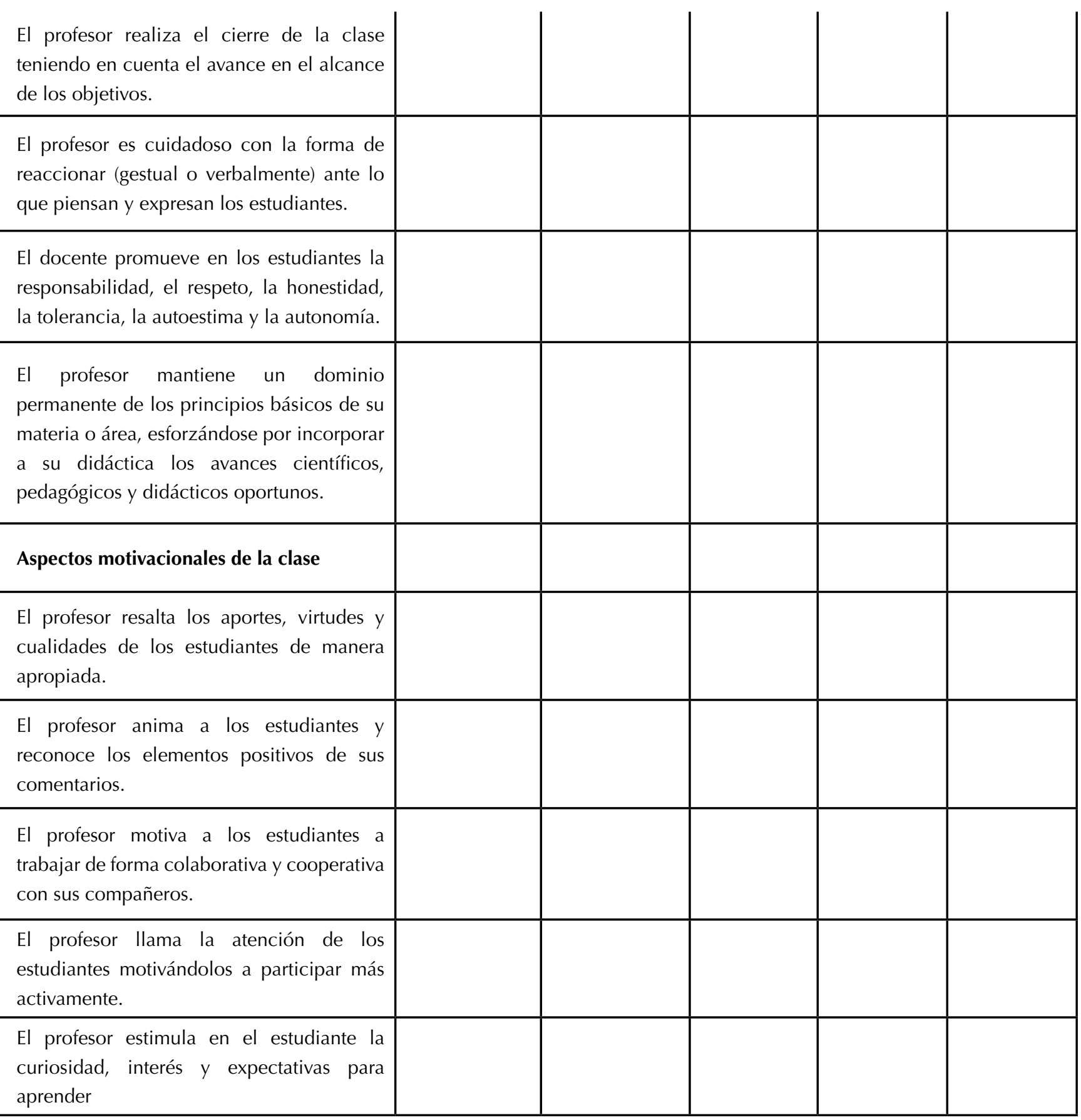

